\title{
CALCULATING STEADY-STATE PROBABILITIES OF QUEUEING SYSTEMS USING HYPEREXPONENTIAL APPROXIMATION
}

\author{
Yuriy Zhernovyi ${ }^{1}$, Bohdan Kopytko ${ }^{2}$ \\ ${ }^{I}$ Ivan Franko National University of Lviv, Lviv, Ukraine \\ ${ }^{2}$ Institute of Mathematics, Czestochowa University of Technology \\ Czestochowa, Poland \\ yu.zhernovyi@lnu.edu.ua,bohdan.kopytko@im.pcz.pl
}

Received: 23 January 2019; Accepted: 25 March 2019

\begin{abstract}
This article proposes an analysis of the results of the application of hyperexponential approximations with parameters of the paradoxical and complex type for calculating the steady-state probabilities of the $\mathrm{G} / \mathrm{G} / \mathrm{n} / \mathrm{m}$ queueing systems with the number of channels $n=1,2$ and 3 . The steady-state probabilities are solutions of a system of linear algebraic equations obtained by the method of fictitious phases. Approximation of arbitrary distributions is carried out using the method of moments. We verified the obtained numerical results using potential method and simulation models, constructed by means of GPSS World.
\end{abstract}

MSC 2010: 60G10,60J28,60K25,93B40

Keywords: non-Markovian queueing system, hyperexponential approximation, complex and paradoxical parameters of distribution

\section{Introduction}

For the study of the non-Markovian process in queueing systems, phase-type distributions are used with exponential distributions of delays in the phases [1-3]. In the case of fixing the number of the phase, the states of the system has a Markov property that makes it possible to represent the transitions between them in the form of a discrete Markov process with continuous time. The order of approximation is the number of retained initial moments of the original distribution.

In many cases, the approximation of an arbitrary distribution by a phase-type distribution using the moments method leads to the fact that the parameters of the approximating distribution are paradoxical (i.e., negative or with probabilities that exceed the boundaries of the interval $[0,1])$ or are complex-valued.

Recently, interest in hyperexponential distribution has increased since its use showed its high performance in solving problems of summation of recurrent flows 
[4], in computing characteristics of queuing systems with impatient customers [5] and Jackson's networks of queueing [6], and also in analyzing stock management systems [7].

Article [3] shows that the use of hyperexponential approximation $\left(H_{k}\right)$ and, in some cases, approximation with the help of the generalized Erlang law $\left(E_{k}\right)$ makes it possible to determine the steady-state probabilities of non-Markovian singlechannel queuing systems with high accuracy.

The initial moments of an $H_{k}$-distribution are calculated by simpler formulas than the moments of an $E_{k}$-distribution and allow for the equalization of $2 k-1$ moments in approximating an arbitrary distribution (for an $E_{k}$-distribution, only $k$ moments can be equalized); therefore, the approximation using an $H_{k}$-distribution is much more efficient. To find parameters of the $H_{k}$-approximation of a certain distribution it is sufficient to solve the system of equations of the moments method. Roots of this system are complex-valued or paradoxical for the values $V<1$ of the variation coefficient, but in most cases, as a result of summation of probabilities of microstates, their complex-valued and paradoxical parts are annihilated.

The purpose of the paper is to analyze the peculiarities of applying hyperexponential distributions with both real and paradoxical and complex-valued parameters for the approximate calculation of steady-state probabilities of $\mathrm{G} / \mathrm{G} / \mathrm{n} / \mathrm{m}$ queues for $n=1,2$ and 3 by means of their approximation by the $H_{k} / H_{k} / n / m$ queueing systems. In particular, we will focus on clarifying the following issues: the influence of increasing the number of channels $G / G / n / m$ queue on the accuracy of calculating the steady-state distribution of the number of customers; ascertainment of conditions for variation coefficients of distributions, for which satisfactory accuracy of the calculation of steady-state probabilities is achieved (better compared to the results of simulation modeling); the study of the properties of the "function" of the $H_{k}$-distribution in the case of paradoxical or complex-valued the distribution parameters and the effect of its deviation from the true distribution function on the accuracy of the results; finding ways to evaluate the accuracy of the approach steady-state distribution of the $H_{k} / H_{k} / n / m$ queue to the true distribution without the need to use simulation models.

\section{Features of approximation by means of $\boldsymbol{H}_{k}$-distributions}

The hyperexponential distribution of order $k$ is a phase-type distribution and provides for choosing one of $k$ alternative phases by a random process. With probability $y_{i}$, the process is at the $i$-th phase and remains in it during an exponentially distributed time with a parameter $\theta_{i}$.

The system of equations of the moments method for approximating the distribution of some random variable $X$ using a random variable $Y_{k}$ distributed by law of $H_{k}$ is of the form 


$$
\sum_{j=1}^{k} \frac{y_{j}}{\theta_{j}^{i}}=\frac{m_{i}}{i !}, \quad 0 \leq i \leq 2 k-1,
$$

where $m_{i}=E\left(X^{i}\right)$ is the initial moment of order $i$ of the random variable $X$. The dependence of the nature of the roots of the system (1) on the values of the variation coefficient $V$ for the original gamma distributions and Weibull distributions is described in [3].

Using equation (1), we find out how the $H_{k}$-distribution parameters change, if instead of the distribution of a random variable $X$ it is necessary to approximate the distribution of the random variable $\tilde{X}=C X$, where $C=$ const. Since $E\left(\tilde{X}^{i}\right)=E\left((C X)^{i}\right)=C^{i} m_{i}$, equations (1) can be rewritten as $\sum_{j=1}^{k} \frac{y_{j}}{\left(\theta_{j} / C\right)^{i}}=\frac{m_{i}}{i !}$, $0 \leq i \leq 2 k-1$. Consequently, if the parameters $y_{j}$ and $\theta_{j}$ of $H_{k}$-approximation of the distribution of the random variable $X$ are known, then the parameters $\tilde{y}_{j}$ and $\tilde{\theta}_{j}$ of $H_{k}$-approximation of the distribution of the random variable $\tilde{X}=C X$ can be obtained by formulas

$$
\tilde{y}_{j}=y_{j}, \quad \tilde{\theta}_{j}=\theta_{j} / C, \quad 1 \leq j \leq k .
$$

The transformation $\tilde{X}=C X$ does not change the form of the law of gamma distributions and Weibull distributions, only the scale parameter changes: $\tilde{\beta}=C \beta$. Since, in addition, the variation coefficient $V$ of any distribution is invariant with respect to the $\tilde{X}=C X$ transformation, in order to determine the parameters of $H_{k}$-approximation for the Weibull distribution and for the gamma distribution with a given coefficient of variation and with the mean $E(\tilde{X})=C$, as well as for the degenerate distribution (case of $V=0$ ), it is sufficient to solve the system (1) for example, for the case $E(X)=1$, and then use the relation (2).

In the case of complex-valued or paradoxical roots $y_{j}$ and $\theta_{j}$ of the system (1), let us name the function $F_{H_{k}}(t)=1-\sum_{j=1}^{k} y_{j} e^{-\theta_{j} t}(t \geq 0)$ the distribution pseudofunction by law of $H_{k}$. Let us show that a function $F_{H_{k}}(t)$ is a real-valued function if $y_{j}$ and $\theta_{j}(1 \leq j \leq k)$ are roots of the system (1).

In fact, if some of the roots of the system (1) are complex-valued, then they can only be complex conjugate, and all possible cases of alternation of signs before the imaginary unit can be reduced to such two:

$$
\text { 1) } \begin{aligned}
& y_{1}=a+i b, \quad \theta_{1}=\alpha+i \beta ; \quad y_{2}=a-i b, \quad \theta_{2}=\alpha-i \beta ; \\
& \text { 2) } y_{1}=a+i b, \quad \theta_{1}=\alpha-i \beta ; \quad y_{2}=a-i b, \quad \theta_{2}=\alpha+i \beta
\end{aligned}
$$

In each of these cases, the imaginary parts in the expression for $F_{H_{k}}(t)$ are reduced, so the result is the real-valued function: 


$$
\begin{aligned}
& y_{1} e^{-\theta_{1} t}+y_{2} e^{-\theta_{2} t}=2 e^{-\alpha t}(a \cdot \cos (\beta t)+b \cdot \sin (\beta t)) \\
& \text { 2) } y_{1} e^{-\theta_{1} t}+y_{2} e^{-\theta_{2} t}=2 e^{-\alpha t}(a \cdot \cos (\beta t)-b \cdot \sin (\beta t))
\end{aligned}
$$

The absolute deviation of the function of distribution by law $G$ from a function $F_{H_{k}}(t)$, whose parameters are roots of the system (1), we will evaluate with the help of the integral $\Delta_{k}(F)=\int_{0}^{\infty}\left|F_{H_{k}}(t)-F_{G}(t)\right| d t$, where $F_{G}(t)$ is the probability distribution function by law $G$.

\begin{tabular}{|c|c|c|c|c|c|}
\hline Distribution name & $\Delta_{2}(F)$ & $\Delta_{3}(F)$ & $\Delta_{4}(F)$ & $\Delta_{5}(F)$ & $\Delta_{6}(F)$ \\
\hline$D$ & 0.3660 & 0.2615 & 0.2098 & 0.1801 & 0.1560 \\
\hline$U[0,2]$ & 0.1139 & 0.0632 & 0.0411 & 0.0295 & 0.0224 \\
\hline$\Gamma(0.001)$ & 0.3629 & 0.2605 & 0.2092 & 0.1773 & 0.1549 \\
\hline$\Gamma(0.1)$ & 0.2864 & 0.1811 & 0.1256 & 0.0894 & 0.0642 \\
\hline$\Gamma(0.2)$ & 0.2121 & 0.1042 & 0.0519 & 0.0250 & 0.0114 \\
\hline$\Gamma(0.3)$ & 0.1436 & 0.0479 & 0.0145 & 0.0038 & 0.0008 \\
\hline$\Gamma(0.4)$ & 0.0867 & 0.0167 & 0.0023 & 0.0002 & $2.4 \cdot 10^{-6}$ \\
\hline$\Gamma(0.49)$ & 0.0481 & 0.0041 & $5.1 \cdot 10^{-5}$ & $3.0 \cdot 10^{-6}$ & $3.8 \cdot 10^{-7}$ \\
\hline$\Gamma(0.6)$ & 0.0167 & 0.0003 & $3.6 \cdot 10^{-5}$ & $6.6 \cdot 10^{-6}$ & $1.7 \cdot 10^{-6}$ \\
\hline$\Gamma(0.7)$ & 0.0007 & $7.2 \cdot 10^{-5}$ & $1.4 \cdot 10^{-5}$ & $3.7 \cdot 10^{-6}$ & $1.2 \cdot 10^{-6}$ \\
\hline$\Gamma(0.8)$ & 0.0058 & 0.0009 & 0.0002 & $8.0 \cdot 10^{-5}$ & $3.3 \cdot 10^{-5}$ \\
\hline$\Gamma(0.9)$ & 0.0055 & 0.0011 & 0.0003 & 0.0001 & $6.2 \cdot 10^{-5}$ \\
\hline$\Gamma(1.1)$ & 0.0090 & 0.0025 & 0.0009 & 0.0004 & 0.0002 \\
\hline$\Gamma(1.5)$ & 0.0614 & 0.0219 & 0.0101 & 0.0055 & 0.0033 \\
\hline$\Gamma(2)$ & 0.1317 & 0.0529 & 0.0270 & 0.0158 & 0.0102 \\
\hline$\Gamma(3)$ & 0.2416 & 0.1055 & 0.0574 & 0.0356 & 0.0240 \\
\hline$\Gamma(4)$ & 0.3146 & 0.1412 & 0.0787 & 0.0497 & 0.0340 \\
\hline$\Gamma(5)$ & 0.3642 & 0.1655 & 0.0932 & 0.0594 & 0.0409 \\
\hline$\Gamma(10)$ & 0.4733 & 0.2182 & 0.1244 & 0.0801 & 0.0557 \\
\hline$W(0.1)$ & 0.2918 & 0.1863 & 0.1314 & 0.0968 & 0.0729 \\
\hline$W(0.2)$ & 0.2200 & 0.1151 & 0.0652 & 0.0383 & 0.0230 \\
\hline$W(0.3)$ & 0.1540 & 0.0621 & 0.0271 & 0.0123 & 0.0058 \\
\hline$W(0.4)$ & 0.0985 & 0.0291 & 0.0096 & 0.0033 & 0.0012 \\
\hline$W(0.5)$ & 0.0555 & 0.0117 & 0.0028 & 0.0007 & 0.0002 \\
\hline$W(0.6)$ & 0.0253 & 0.0042 & 0.0005 & 0.0002 & $5.0 \cdot 10^{-5}$ \\
\hline$W(0.7)$ & 0.0071 & 0.0026 & 0.0006 & $\infty$ & $6.1 \cdot 10^{-5}$ \\
\hline$W(0.8)$ & 0.0043 & $\infty$ & 0.0004 & 0.0001 & $\infty$ \\
\hline$W(0.9)$ & 0.0049 & 0.0005 & $\infty$ & 0.0001 & $4.8 \cdot 10^{-5}$ \\
\hline$W(0.95)$ & 0.0031 & 0.0005 & 0.0001 & $\infty$ & $3.5 \cdot 10^{-5}$ \\
\hline$W(1.1)$ & 0.0096 & 0.0027 & 0.0010 & 0.0004 & 0.0002 \\
\hline$W(2)$ & 0.1863 & 0.1041 & 0.0672 & 0.0473 & 0.0352 \\
\hline$W(3)$ & 0.3973 & 0.2790 & 0.2170 & 0.1786 & 0.1524 \\
\hline
\end{tabular}

Table 1. Values of the absolute deviation $\Delta_{k}(F)$ for different distributions 
Table 1 gives deviation values of $\Delta_{k}(F)$ for $k=2, \ldots, 6$, calculated by results of approximation of such distributions with means $E(X)=1$ : degenerate distribution with a constant value of 1 (coefficient of variation $V=0$ ), labeled as $D$; uniform distribution on the interval $[0,2](V=0.577)$, labeled as $U[0,2]$; gamma distributions and Weibull distributions with different coefficients of variation $V$, labeled as $\Gamma(V)$ and $W(V)$ respectively. With increasing order of $H_{k}$-distribution, the value of the deviation $\Delta_{k}(F)$ decreases, and with the increase of the coefficient of variation for $V>1$ the deviation increases much faster for the distribution of Weibull compared with the gamma distribution. The bold text allocates the minimum values of $\Delta_{k}(F)$ for each $k$ obtained separately for gamma distributions and Weibull distributions. For distributions $W(0.7), W(0.8), W(0.9)$ and $W(0.95)$ for some values of $\mathrm{k}$ the deviation $\Delta_{k}(F)=\infty$. In each of these cases, one of roots of the system (1) is real, but negative: $\theta_{1}<0$. Therefore, for the corresponding distribution pseudo-function, the limit relation $\lim _{t \rightarrow \infty} F_{H_{k}}(t)=\infty$ is valid.

\section{Numerical results}

Let us study the features of $H_{k}$-approximation on examples of $\mathrm{G} / \mathrm{G} / \mathrm{n} / \mathrm{m}$ queueing systems with the number of channels $n=1,2$ and 3 and with the limit $m=15$ on the queue length. We approximate the $G / G / n / m, M / G / n / m$ and $G / M / n / m$ systems by means of the $H_{k} / H_{k} / n / m, M / H_{k} / n / m$ and $H_{k} / M / n / m$ systems respectively, using the order of approximation $k$ from 2 to 6 . The mean service time in all cases is equal to 1 , the load factor of the system $\rho=0.8$. The following uniform distributions have been used: $U[0,2]$ for service times, and $U[0.25,2.25], U[0.125,1.125]$ and $U[1 / 12,0.75]$ for the times elapsed between two consecutive arrivals in cases $n=1, n=2$ and $n=3$ respectively.

Table 2. Results of the calculation of steady-state characteristics of the $M / G / 1 / 15$ systems with different $G$-distributions

\begin{tabular}{|c|c|c|c|c|c|c|c|}
\hline \multirow{3}{*}{$\begin{array}{c}G \text {-distribution } \\
\text { name }\end{array}$} & \multirow{3}{*}{$\begin{array}{c}\text { Characteristic } \\
\text { name }\end{array}$} & \multicolumn{6}{|c|}{ Method of calculation and values of characteristics } \\
\hline & & \multicolumn{5}{|c|}{ Approximation using $H_{k}$} & \multirow{2}{*}{$\begin{array}{l}\text { Potential } \\
\text { method }\end{array}$} \\
\hline & & $k=2$ & $k=3$ & $k=4$ & $k=5$ & $k=6$ & \\
\hline 1 & 2 & 3 & 4 & 5 & 6 & 7 & 8 \\
\hline \multirow{4}{*}{$D$} & $N$ & 2.3831 & 2.3828 & 2.3828 & 2.3828 & 2.3828 & 2.3828 \\
\hline & $\Delta_{k(P o t)}$ & $6.80 \cdot 10^{-3}$ & $1.27 \cdot 10^{-4}$ & $1.59 \cdot 10^{-6}$ & $1.25 \cdot 10^{-8}$ & $1.63 \cdot 10^{-10}$ & - \\
\hline & $\Delta_{(k, k-1)}$ & - & $6.80 \cdot 10^{-3}$ & $1.28 \cdot 10^{-4}$ & $1.60 \cdot 10^{-6}$ & $1.26 \cdot 10^{-8}$ & - \\
\hline & $\Delta_{(6, k)}$ & $6.80 \cdot 10^{-3}$ & $1.27 \cdot 10^{-4}$ & $1.59 \cdot 10^{-6}$ & $1.26 \cdot 10^{-8}$ & - & - \\
\hline \multirow{4}{*}{$\Gamma(0.1)$} & $N$ & 2.3981 & 2.3977 & 2.3977 & 2.3977 & 2.3977 & 2.3977 \\
\hline & $\Delta_{k(P o t)}$ & $6.53 \cdot 10^{-3}$ & $1.19 \cdot 10^{-4}$ & $1.45 \cdot 10^{-6}$ & $1.10 \cdot 10^{-8}$ & $8.56 \cdot 10^{-11}$ & - \\
\hline & $\Delta_{(k, k-1)}$ & - & 0.0065 & $1.20 \cdot 10^{-4}$ & $1.45 \cdot 10^{-6}$ & $1.11 \cdot 10^{-8}$ & - \\
\hline & $\Delta_{(6, k)}$ & $6.53 \cdot 10^{-3}$ & $1.19 \cdot 10^{-4}$ & $1.45 \cdot 10^{-6}$ & $1.11 \cdot 10^{-8}$ & - & - \\
\hline
\end{tabular}


cont. table 2

\begin{tabular}{|c|c|c|c|c|c|c|c|}
\hline 1 & 2 & 3 & 4 & 5 & 6 & 7 & 8 \\
\hline \multirow{4}{*}{$W(0.1)$} & $N$ & 2.3981 & 2.3977 & 2.3977 & 2.3977 & 2.3977 & 2.3977 \\
\hline & $\Delta_{k(P o t)}$ & $6.55 \cdot 10^{-3}$ & $1.20 \cdot 10^{-4}$ & $1.51 \cdot 10^{-6}$ & $1.82 \cdot 10^{-8}$ & $7.81 \cdot 10^{-9}$ & - \\
\hline & $\Delta_{(k, k-1)}$ & - & $6.55 \cdot 10^{-3}$ & $1.21 \cdot 10^{-4}$ & $1.51 \cdot 10^{-6}$ & $1.20 \cdot 10^{-8}$ & - \\
\hline & $\Delta_{(6, k)}$ & $6.55 \cdot 10^{-3}$ & $1.20 \cdot 10^{-4}$ & $1.51 \cdot 10^{-6}$ & $1.20 \cdot 10^{-8}$ & - & - \\
\hline \multirow{4}{*}{$\Gamma(0.49)$} & $N$ & 2.7288 & 2.7286 & 2.7286 & 2.7286 & 2.7286 & 2.7286 \\
\hline & $\Delta_{k(P o t)}$ & $2.12 \cdot 10^{-3}$ & $1.16 \cdot 10^{-5}$ & $6.99 \cdot 10^{-9}$ & $1.56 \cdot 10^{-8}$ & $2.81 \cdot 10^{-7}$ & - \\
\hline & $\Delta_{(k, k-1)}$ & - & $2.12 \cdot 10^{-3}$ & $1.16 \cdot 10^{-5}$ & $1.97 \cdot 10^{-8}$ & $2.65 \cdot 10^{-7}$ & - \\
\hline & $\Delta_{(6, k)}$ & $2.12 \cdot 10^{-3}$ & $1.17 \cdot 10^{-5}$ & $2.81 \cdot 10^{-7}$ & $2.65 \cdot 10^{-7}$ & - & - \\
\hline \multirow{4}{*}{$W(0.5)$} & $N$ & 2.7436 & 2.7433 & 2.7433 & 2.7433 & 2.7433 & 2.7433 \\
\hline & $\Delta_{k(P o t)}$ & $2.62 \cdot 10^{-3}$ & $4.53 \cdot 10^{-5}$ & $1.04 \cdot 10^{-6}$ & $4.65 \cdot 10^{-7}$ & $4.62 \cdot 10^{-7}$ & - \\
\hline & $\Delta_{(k, k-1)}$ & - & $2.63 \cdot 10^{-3}$ & $4.53 \cdot 10^{-5}$ & $7.01 \cdot 10^{-7}$ & $9.05 \cdot 10^{-9}$ & - \\
\hline & $\Delta_{(6, k)}$ & $2.62 \cdot 10^{-3}$ & $4.50 \cdot 10^{-5}$ & $6.93 \cdot 10^{-7}$ & $9.05 \cdot 10^{-9}$ & - & - \\
\hline \multirow{4}{*}{$U[0,2]$} & $N$ & 2.8603 & 2.8596 & 2.8596 & 2.8596 & 2.8596 & 2.8596 \\
\hline & $\Delta_{k(P o t)}$ & $5.20 \cdot 10^{-3}$ & $2.04 \cdot 10^{-4}$ & $6.26 \cdot 10^{-6}$ & $1.48 \cdot 10^{-7}$ & $2.45 \cdot 10^{-9}$ & - \\
\hline & $\Delta_{(k, k-1)}$ & - & $5.23 \cdot 10^{-3}$ & $2.06 \cdot 10^{-4}$ & $6.38 \cdot 10^{-6}$ & $1.50 \cdot 10^{-7}$ & - \\
\hline & $\Delta_{(6, k)}$ & $5.20 \cdot 10^{-3}$ & $2.04 \cdot 10^{-4}$ & $6.25 \cdot 10^{-6}$ & $1.50 \cdot 10^{-7}$ & - & - \\
\hline \multirow{4}{*}{$\Gamma(0.7)$} & $N$ & 3.0549 & 3.0549 & 3.0549 & 3.0549 & 3.0549 & 3.0549 \\
\hline & $\Delta_{k(P o t)}$ & $4.37 \cdot 10^{-5}$ & $4.26 \cdot 10^{-7}$ & $6.48 \cdot 10^{-9}$ & $1.15 \cdot 10^{-10}$ & $5.29 \cdot 10^{-11}$ & - \\
\hline & $\Delta_{(k, k-1)}$ & - & $4.36 \cdot 10^{-5}$ & $4.23 \cdot 10^{-7}$ & $6.41 \cdot 10^{-9}$ & $1.46 \cdot 10^{-10}$ & - \\
\hline & $\Delta_{(6, k)}$ & $4.37 \cdot 10^{-5}$ & $4.26 \cdot 10^{-7}$ & $6.49 \cdot 10^{-9}$ & $1.46 \cdot 10^{-10}$ & - & - \\
\hline \multirow{4}{*}{$W(0.7)$} & $N$ & 3.0575 & 3.0574 & 3.0574 & 3.0574 & 3.0574 & 3.0574 \\
\hline & $\Delta_{k(P o t)}$ & $4.70 \cdot 10^{-4}$ & $2.78 \cdot 10^{-5}$ & $4.50 \cdot 10^{-7}$ & $5.75 \cdot 10^{-8}$ & $4.63 \cdot 10^{-8}$ & - \\
\hline & $\Delta_{(k, k-1)}$ & - & $4.76 \cdot 10^{-4}$ & $2.76 \cdot 10^{-5}$ & $4.34 \cdot 10^{-7}$ & $1.88 \cdot 10^{-8}$ & - \\
\hline & $\Delta_{(6, k)}$ & $4.70 \cdot 10^{-4}$ & $2.78 \cdot 10^{-5}$ & $4.23 \cdot 10^{-7}$ & $1.88 \cdot 10^{-8}$ & - & - \\
\hline \multirow{4}{*}{$\Gamma(0.9)$} & $N$ & 3.4191 & 3.4192 & 3.4192 & 3.4192 & 3.4192 & 3.4192 \\
\hline & $\Delta_{k(P o t)}$ & $4.05 \cdot 10^{-4}$ & $1.15 \cdot 10^{-5}$ & $4.24 \cdot 10^{-7}$ & $1.66 \cdot 10^{-8}$ & $6.64 \cdot 10^{-10}$ & - \\
\hline & $\Delta_{(k, k-1)}$ & - & $4.03 \cdot 10^{-4}$ & $1.13 \cdot 10^{-5}$ & $4.11 \cdot 10^{-7}$ & $1.60 \cdot 10^{-8}$ & - \\
\hline & $\Delta_{(6, k)}$ & $4.05 \cdot 10^{-4}$ & $1.15 \cdot 10^{-5}$ & $4.24 \cdot 10^{-7}$ & $1.60 \cdot 10^{-8}$ & - & - \\
\hline \multirow{4}{*}{$W(0.9)$} & $N$ & 3.4214 & 3.4215 & - & 3.4215 & 3.4215 & 3.4215 \\
\hline & $\Delta_{k(P o t)}$ & $4.16 \cdot 10^{-4}$ & $3.24 \cdot 10^{-6}$ & - & $1.92 \cdot 10^{-7}$ & $1.89 \cdot 10^{-7}$ & - \\
\hline & $\Delta_{(k, k-1)}$ & - & $4.15 \cdot 10^{-4}$ & - & $3.12 \cdot 10^{-6}$ & $1.89 \cdot 10^{-8}$ & - \\
\hline & $\Delta_{(6, k)}$ & $4.15 \cdot 10^{-4}$ & $3.13 \cdot 10^{-6}$ & - & $1.89 \cdot 10^{-8}$ & - & - \\
\hline \multirow{4}{*}{$\Gamma(1.1)$} & $N$ & 3.7964 & 3.7963 & 3.7963 & 3.7963 & 3.7963 & 3.7963 \\
\hline & $\Delta_{k(P o t)}$ & $7.97 \cdot 10^{-4}$ & $4.02 \cdot 10^{-5}$ & $2.44 \cdot 10^{-6}$ & $1.57 \cdot 10^{-7}$ & $1.08 \cdot 10^{-8}$ & - \\
\hline & $\Delta_{(k, k-1)}$ & - & $7.82 \cdot 10^{-4}$ & $3.86 \cdot 10^{-5}$ & $2.31 \cdot 10^{-6}$ & $1.50 \cdot 10^{-7}$ & - \\
\hline & $\Delta_{(6, k)}$ & $7.97 \cdot 10^{-4}$ & $4.02 \cdot 10^{-5}$ & $2.44 \cdot 10^{-6}$ & $1.50 \cdot 10^{-7}$ & - & - \\
\hline \multirow{4}{*}{$W(1.1)$} & $N$ & 3.7917 & 3.7915 & 3.7915 & 3.7915 & 3.7915 & 3.7915 \\
\hline & $\Delta_{k(P o t)}$ & $1.13 \cdot 10^{-3}$ & $6.98 \cdot 10^{-5}$ & $5.96 \cdot 10^{-6}$ & $6.42 \cdot 10^{-7}$ & $1.89 \cdot 10^{-7}$ & - \\
\hline & $\Delta_{(k, k-1)}$ & - & $1.11 \cdot 10^{-3}$ & $6.56 \cdot 10^{-5}$ & $5.60 \cdot 10^{-6}$ & $4.99 \cdot 10^{-7}$ & - \\
\hline & $\Delta_{(6, k)}$ & $1.13 \cdot 10^{-3}$ & $4.02 \cdot 10^{-5}$ & $2.44 \cdot 10^{-6}$ & $4.99 \cdot 10^{-7}$ & - & - \\
\hline
\end{tabular}




\begin{tabular}{|c|c|c|c|c|c|c|c|}
\hline 1 & 2 & 3 & 4 & 5 & 6 & 7 & 8 \\
\hline \multirow{4}{*}{$W(2)$} & $N$ & 4.8597 & 4.9240 & 4.9179 & 4.9164 & 4.9167 & 4.9169 \\
\hline & $\Delta_{k(P o t)}$ & 0.0426 & 0.0150 & $6.11 \cdot 10^{-3}$ & $2.86 \cdot 10^{-3}$ & $1.39 \cdot 10^{-3}$ & - \\
\hline & $\Delta_{(k, k-1)}$ & - & 0.0372 & 0.0104 & $3.92 \cdot 10^{-3}$ & $1.66 \cdot 10^{-3}$ & - \\
\hline & $\Delta_{(6, k)}$ & 0.0431 & 0.0146 & $5.43 \cdot 10^{-3}$ & $1.66 \cdot 10^{-3}$ & - & - \\
\hline \multirow{4}{*}{$W(3)$} & $N$ & 4.9465 & 5.2941 & 5.3824 & 5.4042 & 5.4068 & 5.3934 \\
\hline & $\Delta_{k(P o t)}$ & 0.1412 & 0.0695 & 0.0457 & 0.0330 & 0.0252 & - \\
\hline & $\Delta_{(k, k-1)}$ & - & 0.0876 & 0.0357 & 0.0176 & 0.0100 & - \\
\hline & $\Delta_{(6, k)}$ & 0.1414 & 0.0609 & 0.0273 & 0.0100 & - & - \\
\hline \multirow{4}{*}{$\Gamma(4)$} & $N$ & 5.9436 & 6.1125 & 6.1128 & 6.1106 & 6.1107 & 6.1108 \\
\hline & $\Delta_{k(P o t)}$ & 0.0745 & 0.0221 & $7.62 \cdot 10^{-3}$ & $2.82 \cdot 10^{-3}$ & $1.18 \cdot 10^{-3}$ & - \\
\hline & $\Delta_{(k, k-1)}$ & - & 0.0621 & 0.0164 & $5.42 \cdot 10^{-3}$ & $1.96 \cdot 10^{-3}$ & - \\
\hline & $\Delta_{(6, k)}$ & 0.0740 & 0.0218 & $7.12 \cdot 10^{-3}$ & $1.96 \cdot 10^{-3}$ & - & - \\
\hline
\end{tabular}

For $M / G / 1 / m$ systems, we test the obtained results using the potential method [8], which allows us to calculate the steady-state probabilities $p_{j}$ of the presence $j$ customers in a queueing system.

For $G / G / n / m$ systems, the obtained results are verified using simulation models constructed with the help of the GPSS World tools [9]. The results obtained using GPSS World slightly differ from one another for different numbers of library random-number generators used for simulating random variables, i.e., times elapsed between two consecutive arrivals and service times. Therefore, we use averaged results obtained using simulation models with different values of random-numbers generators that take on values of natural numbers from 6 to 10 .

Let us introduce the designation: $N$ is the average number of customers in the queueing system, and

$$
\begin{aligned}
& \Delta_{k(\text { Pot })}=\sum_{j=0}^{16}\left|p_{j(k)}-p_{j(\text { Pot })}\right|, \quad \Delta_{(k, k-1)}=\sum_{j=0}^{n+15}\left|p_{j(k)}-p_{j(k-1)}\right|, \quad \Delta_{(6, k)}=\sum_{j=0}^{n+15}\left|p_{j(6)}-p_{j(k)}\right|, \\
& \Delta_{k(\text { sim })}=\sum_{j=0}^{n+15}\left|p_{j(k)}-p_{j(\text { sim })}\right|, \quad p_{j(\text { sim })}=\frac{1}{5} \sum_{i=6}^{10} p_{j(\text { sim }, i)}, 0 \leq j \leq n+15,2 \leq k \leq 6 .
\end{aligned}
$$

Here $p_{j(P o t)}$ and $p_{j(k)}$ are values of probabilities $p_{j}$ obtained using the potential method and $H_{k}$-approximation respectively $\left(p_{j(P o t)}=p_{j}\right) ; p_{j(s i m)}$ is the average value of probabilities $p_{j(\operatorname{sim}, i)}$, obtained by means of the simulation model using the number $i$ of random-numbers generator for $6 \leq i \leq 10, n$ is number of queueing system channels. Thus, the quantities $\Delta_{k(P o t)}$ and $\Delta_{k(\operatorname{sim})}$ are measures of deviations of the distributions $\left\{p_{j(k)}\right\}$ from distributions $\left\{p_{j(P o t)}\right\}$ and $\left\{p_{j(s i m)}\right\}$ respectively, and the quantities $\Delta_{(k, k-1)}$ and $\Delta_{(6, k)}$ give an opportunity to estimate the deviation of distributions $\left\{p_{j(k)}\right\}$ from distributions $\left\{p_{j(k-1)}\right\}$ and $\left\{p_{j(6)}\right\}$ respectively. 
In Table 2, we have results of the calculation of steady-state characteristics of the $M / G / 1 / 15$ systems with different $G$-distributions. Those values of $\Delta_{k(P o t)}$ and $\Delta_{(6, k)}(2 \leq k \leq 5)$, that are either identical or are at least numbers of the same order, are bolded. Note that only for distributions $\Gamma(0.49), W(0.5)(k=4,5)$ and $W(0.9)(k=5)$ deviations $\Delta_{k(P o t)}$ and $\Delta_{(6, k)}$ are numbers that differ in one order. This means that in most cases we can use values $\Delta_{(6, k)}$ to evaluate the accuracy of the approximation of the distribution $\left\{p_{j(k)}\right\}$ to the true $\left\{p_{j}\right\}$ for $2 \leq k \leq 5$.

The results presented in Table 2 indicate that the values of absolute deviations $\Delta_{k(P o t)}$ and $\Delta_{(6, k)}$ decrease with increasing order of $H_{k}$-distributions in approximations, as well as the values of $\Delta_{(k, k-1)}$, which decrease with increasing of $k$ means that the values of distribution $\left\{p_{j(k)}\right\}$ with each step getting closer to a true distribution $\left\{p_{j}\right\}$. With the growth of the variation coefficient of distributions after the value of $V>1$, as expected taking into account the behavior of deviations $\Delta_{k}(F)$, the values of the absolute deviations $\Delta_{k(P o t)}$ and $\Delta_{(6, k)}$ also increase. For the distribution $W(0.9)$ the deviation $\Delta_{4}(F)=\infty$ and, consequently, the values of ,probabilities” of the distribution $\left\{p_{j(4)}\right\}$ go beyond the interval $[0,1]$.

Table 3. Rating lists of the $G / G / 1 / 15$ systems with $\Gamma(0.7)$-distributions in order of growth of values $\Delta_{(6,2)}, \Delta_{2(\operatorname{sim})}$ and $\Delta_{(6,5)}$ according to the results of calculations of steady-state distributions

\begin{tabular}{|c|c|c|c|c|c|c|}
\hline \multirow{2}{*}{ No. } & \multicolumn{6}{|c|}{ The name and order of the values of characteristics for which the rating list is compiled } \\
\hline & \multicolumn{2}{|c|}{$\Delta_{(6,2)}$} & \multicolumn{2}{|c|}{$\Delta_{2(\operatorname{sim})}$} & \multicolumn{2}{|c|}{$\Delta_{(6,5)}$} \\
\hline 1 & $M / \Gamma(0.7)$ & \multirow{3}{*}{$10^{-5}$} & $\Gamma(0.7) / \Gamma(0.7)$ & \multirow{6}{*}{$10^{-4}$} & $\Gamma(0.7) / M$ & $10^{-11}$ \\
\hline 2 & $\Gamma(0.7) / M$ & & $M / \Gamma(0.7)$ & & $M / \Gamma(0.7)$ & $10^{-10}$ \\
\hline 3 & $\Gamma(0.7) / \Gamma(0.7)$ & & $\Gamma(0.7) / M$ & & $\Gamma(0.7) / \Gamma(0.7)$ & $10^{-9}$ \\
\hline 4 & $W(0.8) / \Gamma(0.7)$ & \multirow{3}{*}{$10^{-4}$} & $\Gamma(0.7) / W(0.8)$ & & $\Gamma(0.7) / W(0.8)$ & $10^{-8}$ \\
\hline 5 & $\Gamma(0.7) / W(0.8)$ & & $W(0.8) / \Gamma(0.7)$ & & $\Gamma(0.7) / W(0.3)$ & \multirow{3}{*}{$10^{-7}$} \\
\hline 6 & $\Gamma(1.1) / \Gamma(0.7)$ & & $\Gamma(1.1) / \Gamma(0.7)$ & & $\overline{\mid \Gamma(0.1) / \Gamma(0.7)}$ & \\
\hline 7 & $\Gamma(0.7) / \Gamma(1.1)$ & \multirow{6}{*}{$10^{-3}$} & $\Gamma(0.7) / \Gamma(1.1)$ & \multirow{6}{*}{$10^{-3}$} & $\Gamma(0.49) / \Gamma(0.7)$ & \\
\hline 8 & $\Gamma(0.7) / \Gamma(0.49)$ & & $\Gamma(0.7) / \Gamma(0.49)$ & & $\Gamma(0.7) / \Gamma(0.49)$ & \multirow{10}{*}{$10^{-6}$} \\
\hline 9 & $\Gamma(0.49) / \Gamma(0.7)$ & & $\Gamma(0.49) / \Gamma(0.7)$ & & $\Gamma(0.001) / \Gamma(0.7)$ & \\
\hline 10 & $U / \Gamma(0.7)$ & & $U / \Gamma(0.7)$ & & $\Gamma(0.7) / \Gamma(0.1)$ & \\
\hline 11 & $\Gamma(0.7) / W(0.3)$ & & $\Gamma(0.7) / W(0.3)$ & & $\begin{array}{l}(0.7) / \Gamma(1.1) \\
\end{array}$ & \\
\hline 12 & $\Gamma(2) / \Gamma(0.7)$ & & $\Gamma(2) / \Gamma(0.7)$ & & $W(0.3) / \Gamma(0.7)$ & \\
\hline 13 & $\Gamma(0.7) / U$ & \multirow{11}{*}{$10^{-2}$} & $\Gamma(0.7) / U$ & \multirow{11}{*}{$10^{-2}$} & $\Gamma(0.7) / \Gamma(0.001)$ & \\
\hline 14 & $W(0.3) / \Gamma(0.7)$ & & $W(0.3) / \Gamma(0.7)$ & & $\Gamma(1.1) / \Gamma(0.7)$ & \\
\hline 15 & $\Gamma(0.7) / \Gamma(0.1)$ & & $\Gamma(0.7) / \Gamma(0.1)$ & & \begin{tabular}{|l}
$W(0.8) / \Gamma(0.7)$ \\
\end{tabular} & \\
\hline 16 & $\Gamma(0.7) / \Gamma(0.001)$ & & $\Gamma(0.7) / \Gamma(0.001)$ & & $\Gamma(0.7) / U$ & \\
\hline 17 & $W(2) / \Gamma(0.7)$ & & $W(2) / \Gamma(0.7)$ & & $U / \Gamma(0.7)$ & \\
\hline 18 & $\Gamma(0.1) / \Gamma(0.7)$ & & $\Gamma(0.1) / \Gamma(0.7)$ & & $\Gamma(2) / \Gamma(0.7)$ & \multirow{2}{*}{$10^{-4}$} \\
\hline 19 & $\Gamma(0.001) / \Gamma(0.7)$ & & $\Gamma(0.001) / \Gamma(0.7)$ & & $\Gamma(0.7) / \Gamma(2)$ & \\
\hline 20 & $\Gamma(0.7) / \Gamma(2)$ & & $\Gamma(0.7) / \Gamma(2)$ & & $W(2) / \Gamma(0.7)$ & \multirow{4}{*}{$10^{-3}$} \\
\hline 21 & $\Gamma(0.7) / W(2)$ & & $\Gamma(0.7) / W(2)$ & & $\Gamma(0.7) / \Gamma(4)$ & \\
\hline 22 & $\Gamma(0.7) / \Gamma(4)$ & & $\Gamma(0.7) / \Gamma(4)$ & & $\Gamma(0.7) / W(2)$ & \\
\hline 23 & $\Gamma(4) / \Gamma(0.7)$ & & $\Gamma(4) / \Gamma(0.7)$ & & $\begin{array}{l}\Gamma(4) / \Gamma(0.7) \\
\end{array}$ & \\
\hline
\end{tabular}


Table 3 gives rating lists of the $G / G / 1 / 15$ systems with $\Gamma(0.7)$-distributions in order of growth of values $\Delta_{(6,2)}, \Delta_{2(\operatorname{sim})}$ and $\Delta_{(6,5)}$ according to the results of calculations of steady-state distributions $\left\{p_{j(k)}\right\}$ and $\left\{p_{j(\operatorname{sim})}\right\}$. The order of values of deviations $\Delta_{(6,2)}, \Delta_{2(\text { sim })}$ and $\Delta_{(6,5)}$ is also indicated. For deviations $\Delta_{(6,2)}$ and $\Delta_{2(\operatorname{sim})}$ the order in the lists and the order of the values almost completely coincide, minor differences are only in the first five positions. Consequently, in order to estimate the deviation of the distribution $\left\{p_{j(2)}\right\}$ from the true one, instead of the deviation $\Delta_{2(\text { sim })}$ from the distribution $\left\{p_{j(\operatorname{sim})}\right\}$ obtained using the simulation model, you can use the value $\Delta_{(6,2)}$. The order of the values of deviations $\Delta_{(6,5)}$ varies from $10^{-11}$ to $10^{-6}$, and only for the last six positions, corresponding to systems with distributions for which the value of the variation coefficient $V \geq 2$, it ranges from $10^{-4}$ to $10^{-3}$. Consequently, taking into account the value of deviations $\Delta_{(6,5)}$, we can state the high accuracy of the approach of steady-state distributions $\left\{p_{j(k)}\right\}(k=5,6)$ to the true distribution for systems with distributions for which the value of the variation coefficient $V<2$.

Comparison of distributions $\left\{p_{j(k)}\right\}$ with distributions $\left\{p_{j(s i m)}\right\}$ obtained using the simulation model, for $k \geq 3$ does not provide objective information about the approach of $\left\{p_{j(k)}\right\}$ to the true distribution, since the minimum values of the deviation of the distribution $\left\{p_{j(\text { sim })}\right\}$ from the true distribution are numbers of the order $10^{-4}$ or $10^{-3}$

Table 4 gives results of calculation of steady-state distributions $\left\{p_{j(k)}\right\}$ for the $G / G / n / 15$ systems with different number of channels $n$. We see that for some systems, increasing the number of channels leads to a slight increase the deviations $\Delta_{(6, k)}$ by at most one order. As in the case of single-channel systems, the values of deviations $\Delta_{(6,2)}$ and $\Delta_{2(\text { sim })}$ are close, and deviations $\Delta_{6(\text { sim })}$ are numbers of order from $10^{-4}$ to $10^{-3}$. The order of values of deviations $\Delta_{(6,5)}$ varies from $10^{-8}$ to $10^{-3}$ and only for the $\Gamma(4) / \Gamma(0.001) / n / 15$ systems $(n=2,3)$ the deviations $\Delta_{6(\operatorname{sim})}$ and $\Delta_{(6,5)}$ are numbers of order $10^{-2}$. If we consider the values of $\Delta_{6(\text { sim })}$ and $\Delta_{(6,5)}$ not bigger than $10^{-1}$, as the criterion of sufficient accuracy of distribution $\left\{p_{j}\right\}$ approximation by means of distribution $\left\{p_{j(6)}\right\}$, then we can assert that this accuracy is achieved for the $G / G / n / m$ systems $(n=1,2,3)$ with distributions $\Gamma(V)$ and $W(V)$, for which the coefficient of variation satisfies inequalities $V \leq 4$ and $V \leq 3$ respectively. 
Table 4. Results of calculations of steady-state distributions $\left\{p_{j(k)}\right\}$ for the $G / G / n / 15$ systems with $n=1,2$ and 3

\begin{tabular}{|c|c|c|c|c|c|c|c|}
\hline $\begin{array}{l}\text { The name } \\
\text { of the system }\end{array}$ & $n$ & $\Delta_{(6,2)}$ & $\Delta_{(6,3)}$ & $\Delta_{(6,4)}$ & $\Delta_{(6,5)}$ & $\Delta_{2(\operatorname{sim})}$ & $\Delta_{6(\operatorname{sim})}$ \\
\hline \multirow{3}{*}{ М/Г(1.1)/n/15 } & 1 & $7.97 \cdot 10^{-4}$ & $4.02 \cdot 10^{-5}$ & $2.44 \cdot 10^{-6}$ & $1.50 \cdot 10^{-7}$ & 0.0011 & 0.0006 \\
\hline & 2 & $1.49 \cdot 10^{-3}$ & $2.39 \cdot 10^{-4}$ & $4.11 \cdot 10^{-5}$ & $6.72 \cdot 10^{-6}$ & 0.0015 & 0.0006 \\
\hline & 3 & $1.63 \cdot 10^{-3}$ & $3.72 \cdot 10^{-4}$ & $9.22 \cdot 10^{-5}$ & $1.92 \cdot 10^{-5}$ & 0.0021 & 0.0007 \\
\hline \multirow{3}{*}{$\Gamma(1.1) / M / n / 15$} & 1 & $2.08 \cdot 10^{-4}$ & $5.20 \cdot 10^{-6}$ & $5.03 \cdot 10^{-7}$ & $4.77 \cdot 10^{-8}$ & 0.0007 & 0.0007 \\
\hline & 2 & $4.50 \cdot 10^{-4}$ & $1.31 \cdot 10^{-5}$ & $6.18 \cdot 10^{-7}$ & $4.56 \cdot 10^{-8}$ & 0.0010 & 0.0007 \\
\hline & 3 & $4.46 \cdot 10^{-4}$ & $1.58 \cdot 10^{-5}$ & $8.66 \cdot 10^{-7}$ & $5.32 \cdot 10^{-8}$ & 0.0008 & 0.0006 \\
\hline \multirow{3}{*}{$\Gamma(0.001) / \Gamma(1.1) / n / 15$} & 1 & $4.70 \cdot 10^{-3}$ & $7.91 \cdot 10^{-4}$ & $1.83 \cdot 10^{-4}$ & $5.58 \cdot 10^{-5}$ & 0.0047 & 0.0003 \\
\hline & 2 & $5.69 \cdot 10^{-3}$ & $8.47 \cdot 10^{-4}$ & $1.98 \cdot 10^{-4}$ & $3.97 \cdot 10^{-5}$ & 0.0056 & 0.0003 \\
\hline & 3 & $4.96 \cdot 10^{-3}$ & $5.57 \cdot 10^{-4}$ & $2.74 \cdot 10^{-4}$ & $6.81 \cdot 10^{-5}$ & 0.0056 & 0.0010 \\
\hline \multirow{3}{*}{$\Gamma(1.1) / \Gamma(0.001) / n / 15$} & 1 & $4.54 \cdot 10^{-3}$ & $6.41 \cdot 10^{-4}$ & $8.85 \cdot 10^{-5}$ & $1.19 \cdot 10^{-5}$ & 0.0044 & 0.0004 \\
\hline & 2 & 0.0146 & $3.24 \cdot 10^{-3}$ & $3.67 \cdot 10^{-4}$ & $1.19 \cdot 10^{-4}$ & 0.0148 & 0.0005 \\
\hline & 3 & 0.0152 & $6.41 \cdot 10^{-3}$ & $1.21 \cdot 10^{-3}$ & $2.85 \cdot 10^{-4}$ & 0.0157 & 0.0006 \\
\hline \multirow{3}{*}{$U / \Gamma(1.1) / n / 15$} & 1 & $2.07 \cdot 10^{-3}$ & $2.95 \cdot 10^{-4}$ & $3.93 \cdot 10^{-5}$ & $1.57 \cdot 10^{-5}$ & 0.0018 & 0.0004 \\
\hline & 2 & $3.40 \cdot 10^{-3}$ & $3.79 \cdot 10^{-4}$ & $8.28 \cdot 10^{-5}$ & $1.74 \cdot 10^{-5}$ & 0.0036 & 0.0005 \\
\hline & 3 & $3.05 \cdot 10^{-3}$ & $5.00 \cdot 10^{-4}$ & $1.57 \cdot 10^{-4}$ & $2.71 \cdot 10^{-5}$ & 0.0032 & 0.0004 \\
\hline \multirow{3}{*}{$\Gamma(1.1) / U / n / 15$} & 1 & $3.51 \cdot 10^{-3}$ & $2.52 \cdot 10^{-4}$ & $3.40 \cdot 10^{-5}$ & $9.51 \cdot 10^{-6}$ & 0.0034 & 0.0003 \\
\hline & 2 & $6.68 \cdot 10^{-3}$ & $1.46 \cdot 10^{-3}$ & $2.54 \cdot 10^{-4}$ & $5.45 \cdot 10^{-5}$ & 0.0069 & 0.0015 \\
\hline & 3 & $7.21 \cdot 10^{-3}$ & $1.97 \cdot 10^{-4}$ & $6.07 \cdot 10^{-4}$ & $2.22 \cdot 10^{-4}$ & 0.0075 & 0.0007 \\
\hline \multirow{3}{*}{$\Gamma(0.7) / \Gamma(1.1) / n / 15$} & 1 & $1.18 \cdot 10^{-3}$ & $7.76 \cdot 10^{-5}$ & $1.43 \cdot 10^{-5}$ & $1.43 \cdot 10^{-6}$ & 0.0014 & 0.0004 \\
\hline & 2 & $1.83 \cdot 10^{-3}$ & $3.43 \cdot 10^{-4}$ & $5.93 \cdot 10^{-5}$ & $8.85 \cdot 10^{-6}$ & 0.0019 & 0.0006 \\
\hline & 3 & $1.87 \cdot 10^{-3}$ & $4.69 \cdot 10^{-4}$ & $1.23 \cdot 10^{-4}$ & $2.56 \cdot 10^{-5}$ & 0.0020 & 0.0007 \\
\hline \multirow{3}{*}{$\Gamma(1.1) / \Gamma(0.7) / n / 15$} & 1 & $4.96 \cdot 10^{-4}$ & $8.41 \cdot 10^{-5}$ & $1.35 \cdot 10^{-5}$ & $1.87 \cdot 10^{-6}$ & 0.0008 & 0.0007 \\
\hline & 2 & $9.62 \cdot 10^{-4}$ & $4.97 \cdot 10^{-5}$ & $6.69 \cdot 10^{-6}$ & $6.69 \cdot 10^{-7}$ & 0.0012 & 0.0007 \\
\hline & 3 & $8.93 \cdot 10^{-4}$ & $5.52 \cdot 10^{-5}$ & $4.41 \cdot 10^{-6}$ & $1.91 \cdot 10^{-6}$ & 0.0011 & 0.0007 \\
\hline \multirow{3}{*}{$\Gamma(1.1) / \Gamma(1.1) / n / 15$} & 1 & $7.85 \cdot 10^{-4}$ & $5.88 \cdot 10^{-5}$ & $8.86 \cdot 10^{-6}$ & $1.68 \cdot 10^{-6}$ & 0.0009 & 0.0005 \\
\hline & 2 & $1.64 \cdot 10^{-3}$ & $2.26 \cdot 10^{-4}$ & $3.89 \cdot 10^{-5}$ & $6.53 \cdot 10^{-6}$ & 0.0020 & 0.0009 \\
\hline & 3 & $1.84 \cdot 10^{-3}$ & $3.54 \cdot 10^{-4}$ & $8.57 \cdot 10^{-5}$ & $1.86 \cdot 10^{-5}$ & 0.0022 & 0.0006 \\
\hline \multirow{3}{*}{$\Gamma(4) / \Gamma(1.1) / n / 15$} & 1 & 0.0831 & 0.0231 & $8.83 \cdot 10^{-3}$ & $2.64 \cdot 10^{-3}$ & 0.0828 & 0.0020 \\
\hline & 2 & 0.0812 & 0.0238 & $8.83 \cdot 10^{-3}$ & $2.63 \cdot 10^{-3}$ & 0.0810 & 0.0020 \\
\hline & 3 & 0.0791 & 0.0234 & $8.47 \cdot 10^{-3}$ & $2.55 \cdot 10^{-3}$ & 0.0788 & 0.0019 \\
\hline \multirow{3}{*}{$\Gamma(1.1) / \Gamma(4) / n / 15$} & 1 & 0.0697 & 0.0208 & $6.84 \cdot 10^{-3}$ & $1.89 \cdot 10^{-3}$ & 0.0706 & 0.0016 \\
\hline & 2 & 0.1166 & 0.0379 & 0.0146 & $4.61 \cdot 10^{-3}$ & 0.1172 & 0.0043 \\
\hline & 3 & 0.1269 & 0.0520 & 0.0199 & $6.69 \cdot 10^{-3}$ & 0.1282 & 0.0080 \\
\hline \multirow{3}{*}{$\Gamma(4) / \Gamma(0.001) / n / 15$} & 1 & 0.1153 & 0.0336 & 0.0146 & $5.25 \cdot 10^{-3}$ & 0.1125 & 0.0045 \\
\hline & 2 & 0.1610 & 0.0770 & 0.0443 & 0.0231 & 0.1894 & 0.0351 \\
\hline & 3 & 0.1618 & 0.0916 & 0.0568 & 0.0238 & 0.2001 & 0.0506 \\
\hline \multirow{3}{*}{$\Gamma(0.001) / \Gamma(4) / n / 15$} & 1 & 0.1150 & 0.0203 & $7.89 \cdot 10^{-3}$ & $2.52 \cdot 10^{-3}$ & 0.1138 & 0.0028 \\
\hline & 2 & 0.1343 & 0.0441 & 0.0173 & $5.11 \cdot 10^{-3}$ & 0.1331 & 0.0029 \\
\hline & 3 & 0.1284 & 0.0581 & 0.0240 & $7.73 \cdot 10^{-3}$ & 0.1280 & 0.0059 \\
\hline
\end{tabular}


Let $V_{1}$ and $V_{2}$ denote the variation coefficients of the times elapsed between two consecutive arrivals and the service times of the $G / G / n / m$ system. For queueing systems with distributions having small coefficients of variation, namely, the condition $V_{1}+V_{2} \leq 0.6$ is fulfilled, a significant part of the probabilities of distribution $\left\{p_{j}\right\}$ is less than $10^{-5}$, that is the distribution has a "tail", which, as the calculation showed, may lead to the appearance of pseudo-probabilities in distributions $\left\{p_{j(k)}\right\}$.

\section{Conclusions}

If for variation coefficients of distributions of the service times and the times elapsed between two consecutive arrivals, conditions $V_{1}+V_{2}>0.6$ and $\max \left\{V_{1}, V_{2}\right\}<2$ are fulfilled, then the application of hyperexponential approximation allows us to calculate steady-state probabilities of non-Markovian queueing systems with high accuracy (higher than in the case of using simulation models). These probabilities are solutions of a system of linear algebraic equations obtained by the method of fictitious phases. To find parameters of the $H_{k}$-approximation of a certain distribution with a given coefficient of variation, it is sufficient to solve the system of equations of the moments method only for the case of any one given mean value of this distribution since roots of the equations of the moments method are invariant with respect to the scale transformation. For the values $V<1$ of the variation coefficient, these roots are complex-valued but the corresponding pseudofunction of the distribution by law $H_{k}$ is a real-valued function, and its possible unbounded growth only in some cases leads to the impossibility of obtaining a real distribution $\left\{p_{j(k)}\right\}$ which is close to the true distribution. Computing deviations $\Delta_{(k, k-1)}$ and $\Delta_{(6, k)}$ allows us to track the accuracy of approaching distributions $\left\{p_{j(k)}\right\}$ to the true distribution without the need to use simulation models.

An inversely proportional dependence between values of deviations $\Delta_{k}(F)$ (see Table 1) and the accuracy of the calculations of distributions $\left\{p_{j(k)}\right\}$ exists but it weakens with increasing $k$. For distributions $W(0.7), W(0.8), W(0.9)$ and $W(0.95)$ for some values of $k$ the deviation $\Delta_{k}(F)=\infty$. For these values of $k$ the probabilities $p_{j(k)}$ can be complex-valued or go beyond the interval $[0,1]$.

\section{References}

[1] Neuts, M.F. (1981). Matrix-geometric Solutions in Stochastic Models. Baltimore: The John's Hopkins University Press.

[2] Ryzhikov, Yu.I. \& Ulanov, A.V. (2016). Application of hyperexponential approximation in the problems of calculating non-Markovian queuing systems. Vestnik of Tomsk State University. Management, Computer Engineering and Informatics, 3(36), 60-65 (in Russian). 
[3] Zhernovyi, Yu.V. (2018). Calculating steady-state characteristics of single-channel queuing systems using phase-type distributions. Cybernetics and Systems Analysis, 54, 5, 824-832.

[4] Ryzhikov, Yu.I. \& Ulanov, A.V. (2015). Application of hyperexponential approximation in problems of summation of flows. Intelligent Technologies in Transport, 4, $34-39$ (in Russian).

[5] Ryzhikov, Yu.I. \& Ulanov, A.V. (2014). Calculation of the M/ $\mathrm{H}_{2} / \mathrm{n}-\mathrm{H}_{2}$ hyperexponential system with customers impatient in the queue. Vestnik of Tomsk State University. Management, Computer Engineering and Informatics, 2(27), 47-53 (in Russian).

[6] Tsitsiashvili, G.Sh. (2016). Synergistic effect in a network with hyperexponential distributions of service times. Vestnik of Tomsk State University. Management, Computer Engineering and Informatics, 1(34), 65-68 (in Russian).

[7] Nazarov, A.A. \& Broner, V.I. (2016). Inventory management system with hyperexponential distribution of resources consumption. Vestnik of Tomsk State University. Management, Computer Engineering and Informatics, 1(34), 43-49 (in Russian).

[8] Bratiychuk, M. \& Borowska, B. (2002). Explicit formulae and convergence rate for the system $M^{\alpha} / G / 1 / N$ as $N \rightarrow \infty$. Stochastic Models, $18,1,71-84$.

[9] Zhernovyi, Yu. (2015). Creating Models of Queueing Systems Using GPSS World. Saarbrücken: LAP Lambert Academic Publishing. 